\title{
Next-generation of recurrent neural network models for cognition
}

\author{
Guangyu Robert Yang ${ }^{1}$ and Manuel Molano-Mazón ${ }^{2}$ \\ ${ }^{1}$ Center for Theoretical Neuroscience, Columbia University \\ ${ }^{2}$ Brain Circuits \& Behavior lab, IDIBAPS, Barcelona, Spain \\ Contact: gyyang.neuro@gmail.com, manuelmolanomazon@gmail.com
}

\section{Highlights}

- The standard approach of training one Recurrent Neural Network (RNN) with one learning algorithm on one cognitive task needs to be revised.

- Multi-area RNNs should be embraced to model different brain regions that process information hierarchically and in parallel.

- RNNs should be trained with multiple algorithms so they can learn at disparate timescales as animals do.

- A set of canonical tasks is needed to pre-train RNNs in order to guide them towards more naturalistic solutions.

\begin{abstract}
Recurrent Neural Networks (RNNs) trained with machine learning techniques on cognitive tasks have become a widely accepted tool for neuroscientists. In this short opinion piece, we discuss fundamental challenges faced by early work of this approach, and recent steps to overcome such challenges and build next-generation RNN models for cognition. We propose several essential questions that practitioners of this approach should address to continue building future generations of RNN models.
\end{abstract}

\section{Main text}

In the past decade, RNNs have been established as a useful tool to model cognition in humans and other animals [1-3]. Their adoption in neuroscience has made it substantially easier to study the neural dynamics associated with complex cognitive tasks and generate new mechanistic hypotheses [4-16]. As in most other neural network approaches, every use of RNN needs to answer questions about the three core ingredients of deep learning [17]: what network architecture(s) to use, which algorithm(s) to train the network with, and what task(s) or datasets to train the network on. The first generation of RNN models in neuroscience are centered 
around a classical paradigm of studying a single recurrent network trained using one algorithm on one cognitive task. Below we summarize the fundamental limit of this classical paradigm.

Using a single-module RNN. Cognition involves many cortical and subcortical areas, and technological advances are allowing neuronal-level recordings from many areas simultaneously $[18,19]$. It will be extremely difficult to capture such multi-area data with a single-area RNN. Although a single RNN may develop multiple sub-modules through training [20], it is in general not easy to map these sub-modules to actual brain areas. Besides using a single module, RNNs from the classical paradigm rely on architecturally homogeneous units (e.g. vanilla RNNs), and therefore struggle at explaining cell-type specific findings.

Using a single training procedure. Most RNNs are trained with a single machine learning algorithm. Yet, the brain is shaped with processes such as evolution, development, learning, and memory that happen at drastically different time scales. The outcomes of these distinct processes are engraved in biological neural circuits through different forms (architecture, plasticity rules, wiring diagrams, etc.) [21-24]. Relying on a single training procedure governed by one learning time scale might bias RNNs towards substantially less-biological solutions. Note that this is a separate issue from the biological implausibility of gradient-based methods [25]. Most biologically plausible learning algorithms will likely struggle to process information at different time scales when used one at a time.

Training with a single task. Cognitive neural circuits are capable of performing many tasks. By restricting the training to a single laboratory task, RNNs are permitted to ignore many important constraints faced by the brain. In comparison, biological cognitive circuits need to stay flexible and capable of performing many different but related computations in a world with rich statistical structures [26]. RNN models trained on a single simplistic laboratory task may solve it in an apparently optimal manner that nevertheless deviates from the biological solutions.

In the following three sections, we briefly review recent efforts to overcome the issues above.

\section{Building RNNs with more realistic architectures}

One critical issue with the first generation of RNN models is the lack of more biological architectures such as distinct brain areas, cortical layers, and cell types. There are at least two reasons for building RNNs with more realistic architectures. First, experimental findings that are 
increasingly area- and/or cell-type-specific are demanding models with corresponding architectures. Second, many biological architectures are highly conserved throughout evolution, and presumably serve important functions. Using more biologically plausible architectures could potentially help us build more powerful machines as well as allow neural networks to find more "natural" solutions to ecologically relevant tasks.

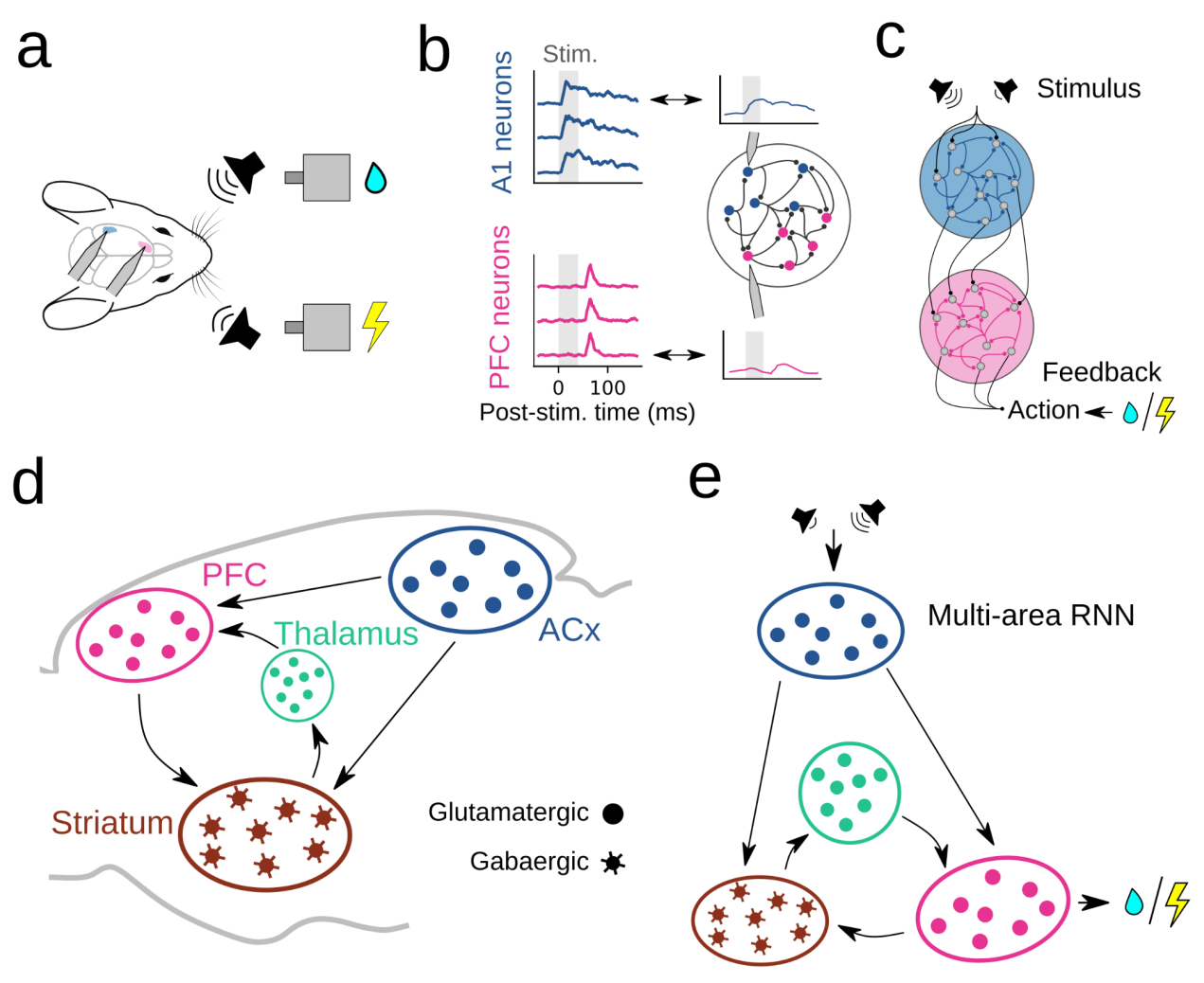

Figure 1. Multi-area RNNs. a) Hypothetical experiment in which a mouse performs a standard auditory two-alternative forced choice (2AFC) task. b) Multi-area RNNs can be built by training different subsets of neurons using neural recordings from distinct brain regions. In the example, recordings from the primary auditory cortex (A1) and prefrontal cortex (PFC) are used to constrain the activity of separate groups of units in the RNN. c) Multi-area RNNs can also be obtained by imposing a predefined connectivity structure with two or more clusters of units organized hierarchically. Here, an RNN simulating A1 (blue) receives sensory inputs and projects to a downstream network (PFC, pink) that makes the final decision. The model can be trained end-to-end to maximize the reward obtained. d-e) Neural circuits are composed of cortical and subcortical areas that have different architectures and neural types (e.g. glutamatergic versus GABAergic) and are connected both hierarchically and in parallel. d) Simplified schematic describing the neural circuit engaged in the auditory task shown in (a): Auditory cortex; thalamus (ventro-anterior and ventro-lateral nuclei); striatum and prefrontal cortex (PFC) [27]. Only projection neurons are shown. e) A network model of the circuit in (d).

Utilizing multiple areas in RNNs is technically straightforward and commonplace in machine learning $[28,29]$. In neuroscience, multi-area RNNs models are increasingly being used to 
address specific questions about how different circuits in the brain interact [30-34]. The main challenge is to have different model areas meaningfully map onto actual brain areas. So far, there are mainly two (nonexclusive) approaches to attempt at a meaningful mapping.

The first approach relies on area-specific neural activity [33]. For example, one may directly train the activity patterns in individual model regions to resemble the neural activity from corresponding biological areas (Fig. 1a,b). This method establishes a rather direct mapping between biological and model areas, even a correspondence between individual neurons of the biological and artificial networks. This approach improves with the amount of experimental data available, and is closely related to the statistical neuroscience approach to multi-area recordings [35].

Another approach is based on area-specific connectivity. For example, one can build a hierarchy of RNNs, where the first area preferentially targets the second, and so on, similar to hierarchical models of the sensory systems (Fig 1c) $[30,32]$. In a hierarchical RNN, modules lower on the hierarchy will have representation/dynamics resembling more the (sensory) inputs, while those higher along the hierarchy will have representations more closely related to the (motor) outputs [30].

Two main challenges remain when building multi-area RNNs. The first one is how to model areas that are not ordered hierarchically, but that process information in parallel, such as dorsal-lateral versus dorsal-medial prefrontal cortex. One possibility is to have different model areas receive qualitatively different inputs [36] and/or generate different kinds of outputs [37]. The second challenge is how to model areas that are architecturally and morphologically distinct from the cortex, such as basal ganglia, thalamus, hippocampus, and cerebellum (Fig. 1d, e). Models containing cortical and subcortical areas have been studied in the classical Connectionist literature [38] and more recently [39,40], but they have not yet been widely combined with cortical RNN models. Subcortical areas are structurally distinct from neocortex (inhibitory projection neurons in basal ganglia, lack of recurrent excitation in thalamus, etc.), but it remains largely unknown which distinctive architectural features are critical to explain the functional role of each subcortical region. 


\section{Moving beyond BPTT with memory and multi-time-scale learning}

Training of RNNs have relied heavily on gradient-based methods (e.g. Stochastic Gradient Descent (SGD)) that compute gradient with back-propagation-through-time (BPTT) [41], which is not biologically plausible partly because it is not causal (information flows backward in time). It is possible to compute the gradient as the network runs forward in time (e.g. RTRL, Real Time Recurrent Learning [42]). However, computing the exact gradient in this manner introduces other non-biological requirements, in particular a $O\left(N^{3}\right)$ memory where $N$ is the number of neurons. In contrast, there are only $O\left(N^{2}\right)$ synaptic variables. Recent work has proposed various biologically plausible approximations to RTRL that reduce the memory requirement to $O\left(N^{2}\right)$ $[43,44]$. These approximate methods generally perform on par with or worse than BPTT at increasing task accuracy during training. The impact of using these approximate methods on the solutions learned in RNNs remains largely unknown.

Besides their biological implausibility, we argue that there are at least two fundamental, interrelated issues of gradient-based methods that can bias the post-training solutions towards less biological ones. The two issues are: (1) difficulty in very long-term credit assignment, and (2) lack of multi-time-scale training.

BPTT suffers from the well known vanishing and exploding gradient problems, making long-term credit assignment hard $[45,46]$. This issue can be alleviated by adopting specialized and less biological architectures, for example LSTMs [47]. However, even with these architectures, BPTT only allows effective credit assignment for thousands of time steps back [48], or about an hour in real time (assuming one second per time step).

We speculate that the difficulty in very long-term credit assignment arises partly because back-propagation — and gradient computation more generally — insists on considering everything that takes place between a cause and its effect before credit assignment. Consider for instance traveling to a conference, and realizing that you forgot to bring the poster days after packing. To assign credit between events separated by days, it would be terribly ineffective to take into account all events that happened in between, as standard learning algorithms do. Similarly, the outcome of the decision made by an animal at a given trial of a laboratory task is the result of events both recent and distant in the past (Fig. 2a). A number of works in machine learning are starting to tackle this issue of very long-term credit assignment [25,49-52]. One 
class of solutions involves storing past events in long-term memory for later retrievals [53], which effectively produces skip connections for gradient computation linking events separated by a long time. Importantly, having a long-term memory component in RNNs (Fig. 2b) could not only improve very long-term credit assignment, it may further shape the solutions found by the training algorithm, biasing the network from statistics-based solutions towards memory-based ones.

The second challenge with BPTT is that it relies on a single learning time scale dictated by the overall learning rate [52]. Even with adaptive learning rate methods such as Adam [54], the overall learning rate still determines on average how fast synaptic weights can change. This fundamental issue manifests itself in many applications. The lack of fast time scale learning prevents synaptic weights from changing rapidly, making it difficult to learn with a few examples (few-shot learning problem). The lack of slow time scale learning prevents synapses from staying stable for a sufficiently long time, a partial cause to the catastrophic forgetting of previous knowledge (continual learning problem) [55].

The general machine learning solution to this challenge is to introduce multiple learning processes of different time scales, often in the form of meta-learning (namely learning-to-learn) [56]. Currently, there is a great diversity of methods, mostly adapted from machine learning, to include multi-time-scale learning into RNNs (Fig. 2b). Some studies use two sets of connection weights subject to fast and slow learning respectively [57-59]. Alternatively, other approaches rely on slow meta-learning to change synaptic weights so that network dynamics can perform fast learning [60] or to discover plausible synaptic plasticity rules [61,62]. In many continual learning applications, the same synapse can be subject to faster or slower learning depending on whether it is important for previously learned tasks [55]. Finally, the above approaches can be combined with genetic algorithms $[62,63]$ that emulate evolution and allow for exploration of different neural architectures, wiring rules or learning algorithms (Fig. 2b). To build better RNN models of brains, it is necessary to identify, potentially from the vast number of existing methods, multi-time-scale learning rules similar to the ones used in the brain as well as the variables that are subject to each learning process. 
a
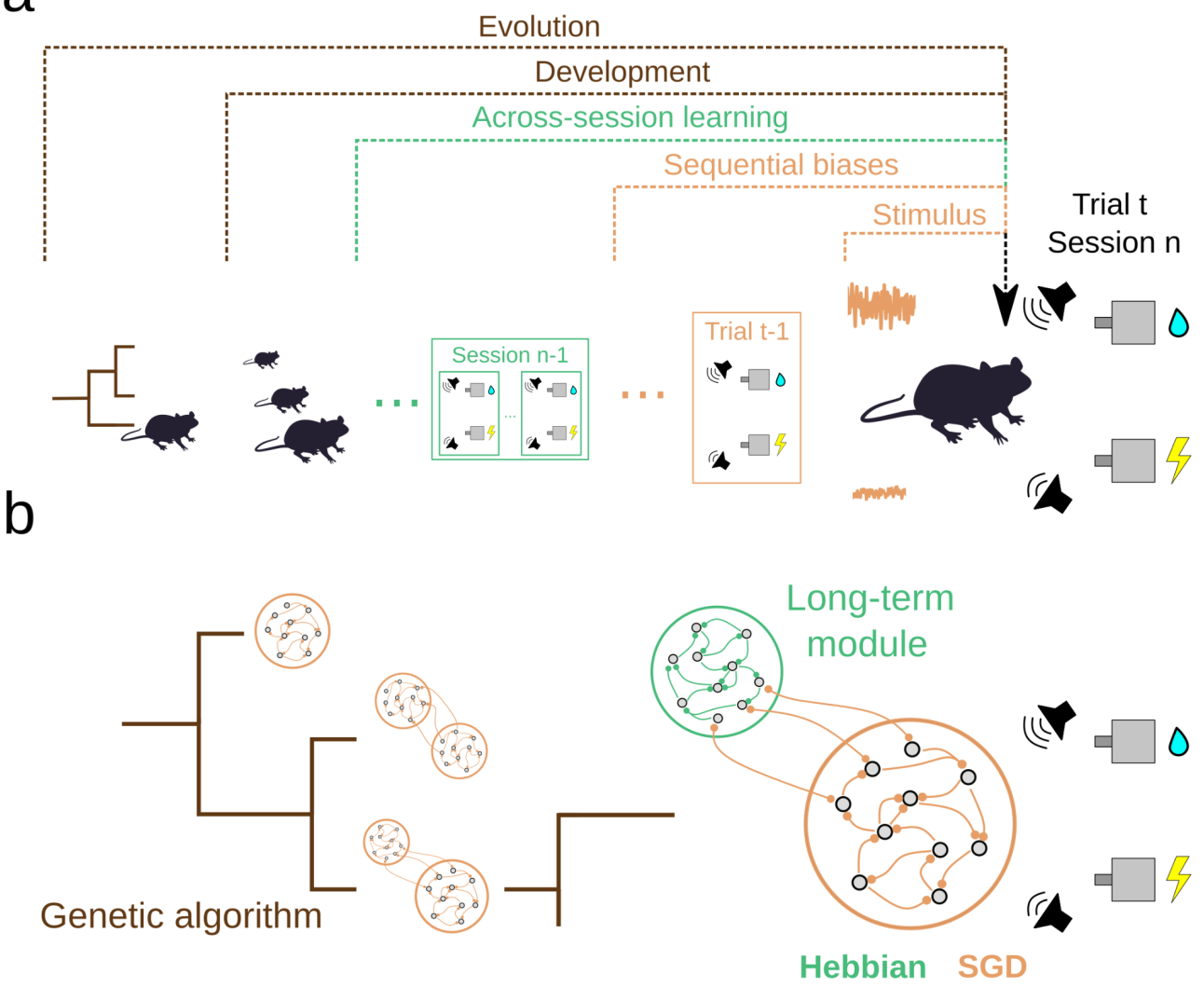

Figure 2. Long-term memory and multi-timescale processing in RNNs. a) The decision of a mouse performing a 2AFC task depends on events happening at wildly disparate time scales, from millions of years (evolution), to years (development), to days (across-session learning), to minutes (sequential biases), and to hundreds of milliseconds (accumulation of stimulus evidence). b) To a first approximation, these different time scales can be implemented by combining genetic algorithms (left) that select the architecture, with long-term memory modules (right, small green network) that store relevant past events, and different types of connections that learn at distinct paces, potentially using different mechanisms (e.g. Hebbian and stochastic gradient descent (SGD)).

\section{Pre-training in ecologically relevant environments}

Most RNN models in neuroscience are trained on just one or a few environments, often abstractions of the already simplified tasks used in experimental studies. Yet, flexibility is a hallmark of cognition, with cognitive circuits shared across many different tasks (Fig. 3a) [64]. Training RNNs to perform many tasks is therefore necessary to understand how different computations are supported by the same network (Fig. 3b) [20,60,65,66], and how tasks can be learned sequentially in the same network [55]. Even if one is interested in understanding the neural mechanism that solves a single task, we argue that it may be appropriate to first train RNNs to perform many other naturalistic tasks, so the neural mechanisms found by the RNNs are similar to those used by biological networks. 

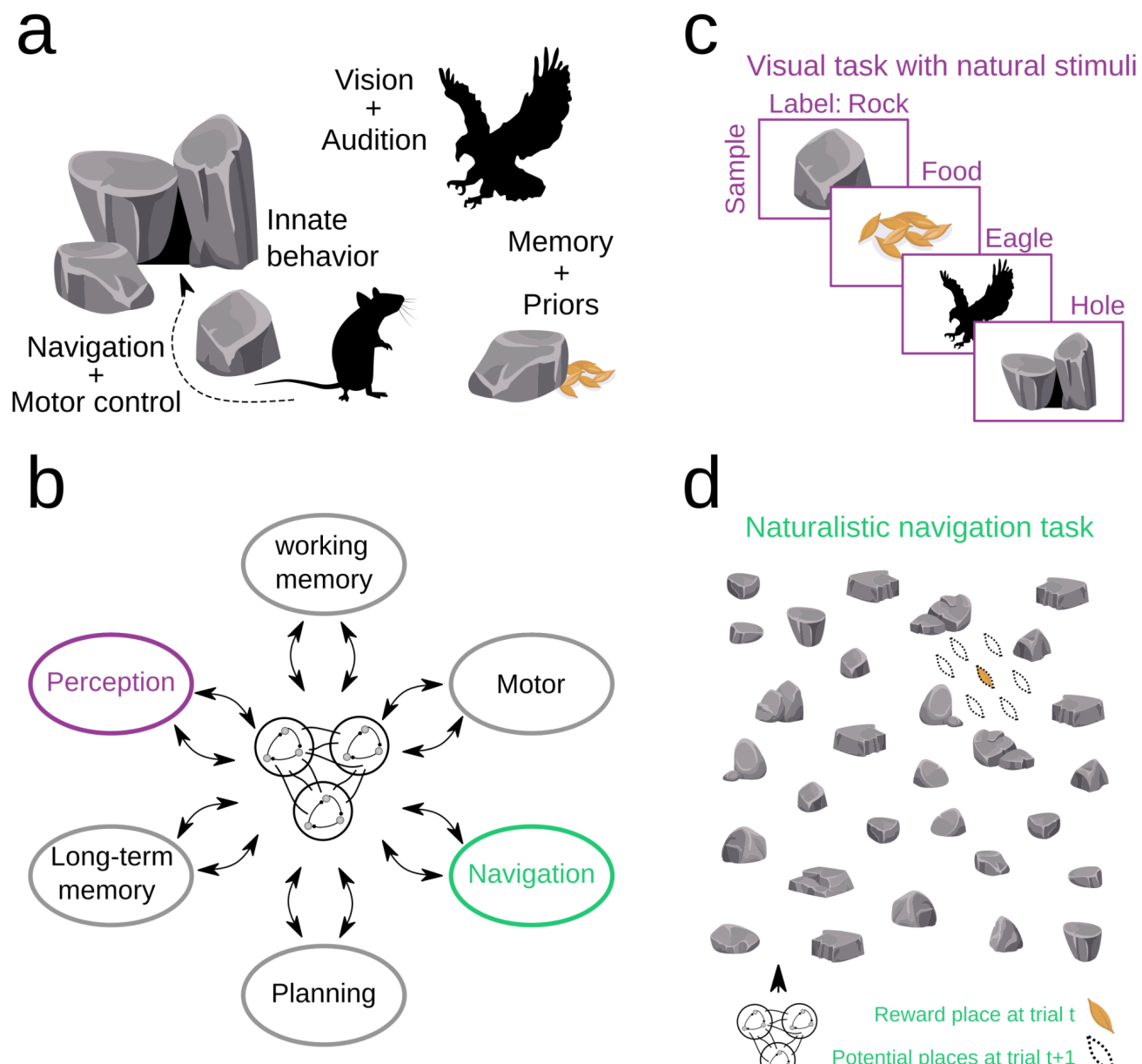

d

Naturalistic navigation task

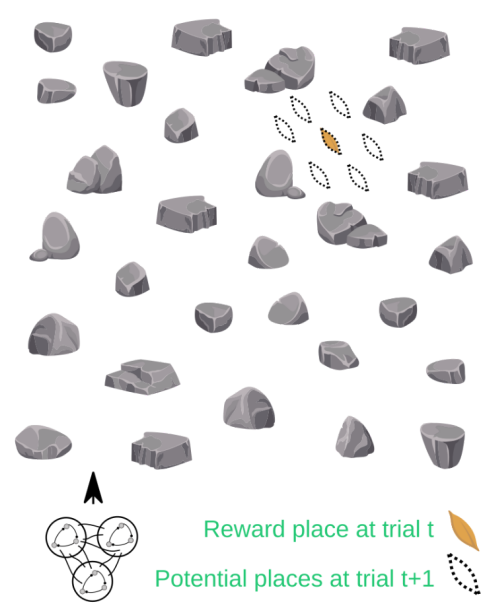

Figure 3. Training RNNs on multiple naturalistic tasks. a) Cartoon showing the range of skills a mouse needs to survive in nature: processing sensory information to detect and identify approaching animals (e.g. an eagle); escaping from predators by quickly hiding; finding food (e.g. barley seeds) using previous experience; etc. To tackle these challenges, mice possess innate behaviors and implicit priors: a tendency to look for small dark holes [67] and to escape from looming visual stimuli [68], and the implicit prior that rewarding places tend to stay rewarding over time (win-stay strategy [69]). b) In order to fully model the mouse brain, a neural network should be pre-trained on a core set of cognitive tasks. c-d) Tasks should aim to emulate the natural habitat of animals with high-dimensional inputs (c) and the spatio-temporal statistics of natural environments (e.g. persistence) (d), so as to develop similar priors that guide and constrain neural computations in the brain.

Humans and other animals arrive at the laboratory with fundamental priors built by evolution and development to behave in a world with specific spatio-temporal statistics (Fig. 3a) $[21,22,70-72]$. Typically, RNNs are trained exclusively on the cognitive task(s) of interest, largely sidestepping the process of evolution and development. By ignoring these constraints, an RNN is permitted to change all possible connections to excel at the only task it is trained on. With a powerful enough training algorithm, the network can learn an almost-optimal solution, which 
nevertheless deviates substantially from biological solutions to the same task. For example, a typical RNN trained on a single task will encode only task-relevant variables, while neural activity in the brain has been shown to be driven primarily by task-irrelevant variables [73].

How to address this issue of the lack of development in RNNs? A number of researchers have suggested that it is necessary to pre-train RNNs with ecologically relevant environments (Fig. $3 c$, d) $[70,72,74]$. Pre-training is particularly urgent for building RNN models of human cognition, since human subjects enter a lab with considerable prior knowledge, but it can be equally relevant for RNN models of non-human animals. The central issue is that little is known about what tasks are appropriate or essential for pre-training [17,75]. In modeling sensory systems using feedforward neural networks, pre-training commonly uses standardized large sensory datasets (e.g. ImageNet [76]). No equivalent datasets or collections of tasks currently exist for RNN models of cognition.

We suggest that establishing and refining a set of core tasks for pre-training RNNs will be instrumental to the next-generation of RNN models. This core set of tasks should address the essential cognitive capabilities of animals and the demands imposed by their habitats (Fig. 3). A number of task collections have been developed [77], but it remains to be seen whether pre-training on these tasks is sufficient to explain the flexibility of cognitive systems.

\section{Is biological realism always the answer?}

So far, we summarized three directions for what we believe should be the next generation of RNN models of cognition. All directions involve making RNNs more biological and closer to brains in architectures, learning, and behavior. However, one may correctly argue that a key distinctive feature of RNN models, compared to more classical models of cognition, is a stronger emphasis on functionality (top-down approach) and a relative de-emphasis on biologically realistic components (bottom-up approach). We argue that this core distinction of RNN models should be maintained, and we should continue to pursue computational models that can display complex behaviors. However, we also believe that introducing the right kind of biological realism will enhance functionality of RNN models, especially in more naturalistic environments. After all, the brain is both biological and functional. 


\section{Future generations of RNNs: Answering how architecture, learning, and tasks interact}

We have discussed emerging efforts to design the next-generation of RNN models for cognition. To our knowledge, most recent work focused on innovating in one of the three core components of RNN design: architecture, learning, and tasks. Future generations of RNN models will need to answer how these three aspects interact with one another. For example, when an RNN contains multiple areas and multiple learning mechanisms of different time scales, how should the learning mechanisms be distributed across areas? If an RNN is pretrained on many tasks, what learning mechanism should be used for pre-training and what should be used for in-lab learning? In a multi-area RNN undergoing pre-training, which areas or synapses should be pre-trained?

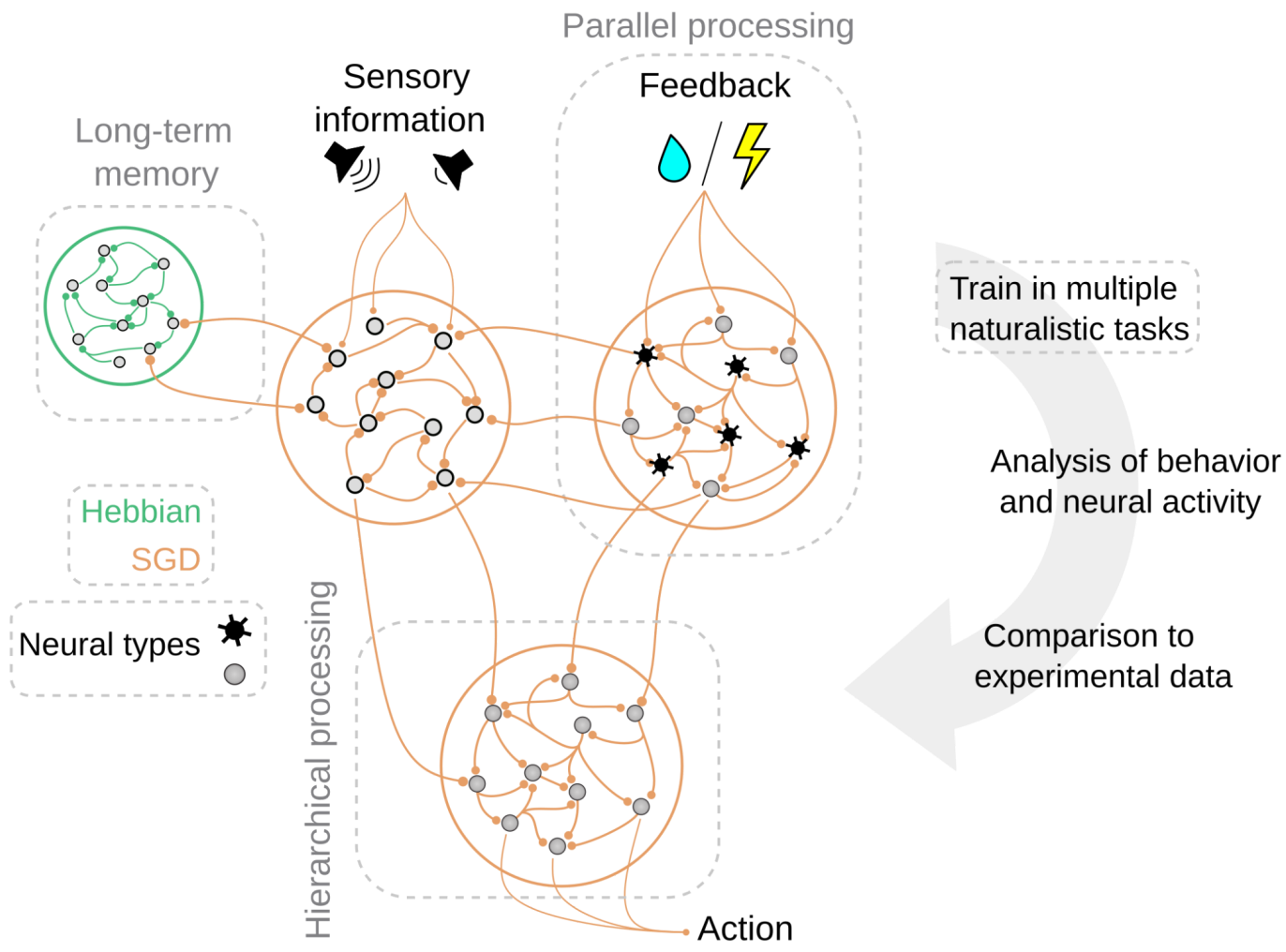

Figure 4. Next-generation of recurrent neural network models for cognition. The next generation of neural networks should incorporate several new functionalities (dashed rectangles) in order to model the capabilities of real brains: parallel and hierarchical multi-area networks, potentially receiving different information (e.g. stimulus versus feedback); long-term memory modules (green network) that store relevant events far in the past; and learning mechanisms that operate across a wide range of time scales. Finally, network models should be trained in multiple tasks that simulate naturalistic environments. 
Answering these questions requires close interactions between theory, modeling, and experiments. We argue that there are so many open questions that it is necessary to speed up exploration by building high-throughput pipelines for developing and testing RNN models [78] (Fig. 4). Large benchmarks are critical for the rapid iteration of deep networks in machine learning. Similar benchmarks are being deployed for neural network models of visual systems [79]. It is reasonable to caution against a blind passion for standard benchmarks. However, to build and verify ever more complex RNN models, it is necessary to test and compare such models on large-scale neural and behavioral datasets, pooling results from across labs and experimental paradigms.

\section{Summary}

While the adoption of RNNs has provided new blood and new options in the toolbox of neuroscientists for modeling cognition, the classical paradigm of RNN modeling awaits major revisions and revolutions on all fronts. All three key ingredients for building an RNN model-network architecture, training algorithm, and task-need to be revamped. Ongoing and future innovations will help us build RNNs that can model experimental results across different areas, layers, and cell types. New multi-time-scale learning rules combined with long-term memory will allow RNNs to both adapt rapidly and maintain knowledge for a long time. RNNs pre-trained on a set of core tasks will lead to networks learning and solving tasks in more biological ways.

\section{Acknowledgments}

GRY is supported by Simons Foundation Junior Fellowship, NSF NeuroNex Award 589 DBI-1707398, and the Gatsby Charitable Foundation. MMM acknowledges support by the Beatriu de Pinós fellowship, Generalitat de Catalunya (2017-BP-00305) and by the Spanish Ministry of Science (RTI2018-099750-B-I00). We are grateful to Joao Barbosa for discussion and feedback. We thank Daniel Duque, Albert Compte, Ling Liang Dong, and Maria Fernanda De La Torre for feedback on the manuscript. We thank Carles Sindreu for his help with figures. 
Highlighted papers:

* Duncker, Lea, Laura Driscoll, Krishna V. Shenoy, Maneesh Sahani, and David Sussillo. 2020. "Organizing Recurrent Network Dynamics by Task-Computation to Enable Continual Learning." Advances in Neural Information Processing Systems 33. https://proceedings.neurips.cc/paper/2020/file/a576eafbce762079f7d1f77fca1c5cc2-Paper. pdf.

The authors proposed a new continual learning rule that separates the neural activity dynamics associated with different tasks into orthogonal subspaces. This learning rule alleviates catastrophical interference in networks trained on multiple tasks sequentially, and allows reusing of existing dynamics motifs when new tasks are learned.

* Kleinman, M., C. Chandrasekaran, and J. C. Kao. 2019. "Recurrent Neural Network Models of Multi-Area Computation Underlying Decision-Making." bioRxiv. https://www.biorxiv.org/content/10.1101/798553v1.abstract

Kleinman and colleagues train multi-area RNNs with constrained connectivity based on Dale's law. They show that in the dorsal premotor cortex (PMd) of monkeys performing a perceptual decision making task, the neural dynamics is reproduced in the last region of a multi-area RNN, but not in a single-area RNN.

* Hung, Chia-Chun, Timothy Lillicrap, Josh Abramson, Yan Wu, Mehdi Mirza, Federico Carnevale, Arun Ahuja, and Greg Wayne. 2019. “Optimizing Agent Behavior over Long Time Scales by Transporting Value." Nature Communications 10 (1): 5223.

The authors demonstrate how a long-term memory module can be used for long-term credit assignment in deep reinforcement learning. Agents learn to predict future rewards using stored memories of past events. Unexpected reward triggers re-valuation of such memories, assigning credit from the present to the potentially distant past.

** Michaels, Jonathan A., Stefan Schaffelhofer, Andres Agudelo-Toro, and Hansjörg Scherberger. 2020. “A Goal-Driven Modular Neural Network Predicts Parietofrontal Neural Dynamics during Grasping." Proceedings of the National Academy of Sciences of the United States of America 117 (50): 32124-35. 
Michaels et al. train 3-area RNNs to produce the muscle dynamics when monkeys are grasping objects with visual guidance. They show that by modelling the modular architecture of the neural circuit engaged in grasping (anterior intraparietal area $\rightarrow$ hand area $\rightarrow$ primary motor cortex) they can replicate the dynamics of the circuit, outperforming alternative models. This allows them to propose mechanistic hypotheses for the neural computations involved in visually guided grasping of objects.

**Andalman, Aaron S., Vanessa M. Burns, Matthew Lovett-Barron, Michael Broxton, Ben Poole, Samuel J. Yang, Logan Grosenick, et al. 2019. "Neuronal Dynamics Regulating Brain and Behavioral State Transitions." Cell 177 (4): 970-85.e20.

This paper trains RNN models to reproduce the activity recorded from the brain of zebrafish using brain-wide imaging. The authors use their model to propose a neural mechanism for the transition from active to passive coping observed in zebrafishes exposed to an unavoidable aversive environment.

** Wang, Jane X., Zeb Kurth-Nelson, Dharshan Kumaran, Dhruva Tirumala, Hubert Soyer, Joel Z. Leibo, Demis Hassabis, and Matthew Botvinick. 2018. "Prefrontal Cortex as a Meta-Reinforcement Learning System." Nature Neuroscience 21 (6): 860-68.

Wang and colleagues use a meta-learning approach to train RNNs with two different time scales. The authors use this framework to suggest that the dopamine system trains the prefrontal cortex (PFC) using a slow reinforcement learning algorithm, allowing the PFC to implement a second, faster learning algorithm based on its recurrent dynamics.

** Yang, Guangyu Robert, Madhura R. Joglekar, H. Francis Song, William T. Newsome, and Xiao-Jing Wang. 2019. "Task Representations in Neural Networks Trained to Perform Many Cognitive Tasks.” Nature Neuroscience 22 (2): 297-306.

This paper shows how networks trained on many related tasks develop a modular structure that allows a compositional task representation. When tasks are trained continually, the networks display mixed task selectivity similar to that of prefrontal neurons of monkeys switching between two different tasks. 


\section{References}

1. Sussillo D. Neural circuits as computational dynamical systems. Curr Opin Neurobiol. 2014;25: 156-163.

2. Barak O. Recurrent neural networks as versatile tools of neuroscience research. Curr Opin Neurobiol. 2017;46: 1-6.

3. Yang GR, Wang X-J. Artificial neural networks for neuroscientists: a primer. Neuron. 2021;109: 739 .

4. Mante V, Sussillo D, Shenoy KV, Newsome WT. Context-dependent computation by recurrent dynamics in prefrontal cortex. Nature. 2013;503: 78-84.

5. Sussillo D, Churchland MM, Kaufman MT, Shenoy KV. A neural network that finds a naturalistic solution for the production of muscle activity. Nat Neurosci. 2015;18: 1025-1033.

6. Carnevale F, de Lafuente V, Romo R, Barak O, Parga N. Dynamic Control of Response Criterion in Premotor Cortex during Perceptual Detection under Temporal Uncertainty. Neuron. 2015;86: 1067-1077.

7. Chaisangmongkon W, Swaminathan SK, Freedman DJ, Wang X-J. Computing by Robust Transience: How the Fronto-Parietal Network Performs Sequential, Category-Based Decisions. Neuron. 2017;93: 1504-1517.e4.

8. Cueva CJ, Wei X-X. Emergence of grid-like representations by training recurrent neural networks to perform spatial localization. arXiv [q-bio.NC]. 2018. Available: http://arxiv.org/abs/1803.07770

9. Wang J, Narain D, Hosseini EA, Jazayeri M. Flexible timing by temporal scaling of cortical responses. Nat Neurosci. 2018;21: 102-110.

10. Remington ED, Narain D, Hosseini EA, Jazayeri M. Flexible Sensorimotor Computations through Rapid Reconfiguration of Cortical Dynamics. Neuron. 2018;98: 1005-1019.e5.

11. Mastrogiuseppe F, Ostojic S. Linking Connectivity, Dynamics, and Computations in Low-Rank Recurrent Neural Networks. Neuron. 2018;99: 609-623.e29.

12. Farrell M, Recanatesi S, Moore T, Lajoie G, Shea-Brown E. Recurrent neural networks learn robust representations by dynamically balancing compression and expansion. bioRxiv. 2019. p. 564476. doi:10.1101/564476

13. Masis JA, Chapman T, Rhee JY, Cox DD, Saxe AM. Rats strategically manage learning during perceptual decision making. bioRxiv. 2020. Available:

https://www.biorxiv.org/content/10.1101/2020.09.01.259911v1.abstract

14. Saxena S, Russo A, Cunningham J, Churchland MM. Motor cortex activity across movement speeds is predicted by network-level strategies for generating muscle activity. 
bioRxiv. 2021. Available:

https://www.biorxiv.org/content/10.1101/2021.02.01.429168v1.abstract

15. Feulner B, Clopath $\mathrm{C}$. Neural manifold under plasticity in a goal driven learning behaviour. PLoS Comput Biol. 2021;17: e1008621.

16. Schaeffer R, Khona M, Meshulam L, Fiete IR, International Brain Laboratory. Reverse-engineering Recurrent Neural Network solutions to a hierarchical inference task for mice. doi:10.1101/2020.06.09.142745

17. Richards BA, Lillicrap TP, Beaudoin P, Bengio Y, Bogacz R, Christensen A, et al. A deep learning framework for neuroscience. Nat Neurosci. 2019;22: 1761-1770.

18. Siegle JH, Jia X, Durand S, Gale S, Bennett C, Graddis N, et al. Survey of spiking in the mouse visual system reveals functional hierarchy. Nature. 2021;592: 86-92.

19. Jun JJ, Steinmetz NA, Siegle JH, Denman DJ, Bauza M, Barbarits B, et al. Fully integrated silicon probes for high-density recording of neural activity. Nature. 2017;551: 232-236.

20. Yang GR, Joglekar MR, Song HF, Newsome WT, Wang X-J. Task representations in neural networks trained to perform many cognitive tasks. Nat Neurosci. 2019;22: 297-306.

21. Cisek P. Resynthesizing behavior through phylogenetic refinement. Atten Percept Psychophys. 2019;81: 2265-2287.

22. Zador AM. A critique of pure learning and what artificial neural networks can learn from animal brains. Nat Commun. 2019;10: 3770.

23. Barabási DL, Beynon T. Complex Computation from Developmental Priors. bioRxiv. 2021. Available: https://www.biorxiv.org/content/10.1101/2021.03.29.437584v1.abstract

24. Koulakov A, Shuvaev S, Zador A. Encoding innate ability through a genomic bottleneck. bioRxiv. 2021. Available:

https://www.biorxiv.org/content/10.1101/2021.03.16.435261v1.abstract

25. Lillicrap TP, Santoro A, Marris L, Akerman CJ, Hinton G. Backpropagation and the brain. Nat Rev Neurosci. 2020;21: 335-346.

26. Gomez-Marin A, Ghazanfar AA. The Life of Behavior. Neuron. 2019;104: 25-36.

27. Chen L, Wang X, Ge S, Xiong Q. Medial geniculate body and primary auditory cortex differentially contribute to striatal sound representations. Nat Commun. 2019;10: 418.

28. Pascanu R, Gulcehre C, Cho K, Bengio Y. How to Construct Deep Recurrent Neural Networks. arXiv [cs.NE]. 2013. Available: http://arxiv.org/abs/1312.6026

29. Schmidhuber J. Learning complex, extended sequences using the principle of history compression. Neural Comput. 1992;4: 234-242.

30. Kleinman M, Chandrasekaran C, Kao JC. Recurrent neural network models of multi-area computation underlying decision-making. bioRxiv. 2019. Available:

https://www.biorxiv.org/content/10.1101/798553v1.abstract 
31. Pinto L, Rajan K, DePasquale B, Thiberge SY, Tank DW, Brody CD. Task-Dependent Changes in the Large-Scale Dynamics and Necessity of Cortical Regions. Neuron. 2019;104: 810-824.e9.

32. Michaels JA, Schaffelhofer S, Agudelo-Toro A, Scherberger H. A goal-driven modular neural network predicts parietofrontal neural dynamics during grasping. Proc Natl Acad Sci U S A. 2020;117: 32124-32135.

33. Andalman AS, Burns VM, Lovett-Barron M, Broxton M, Poole B, Yang SJ, et al. Neuronal Dynamics Regulating Brain and Behavioral State Transitions. Cell. 2019;177: 970-985.e20.

34. Perich MG, Rajan K. Rethinking brain-wide interactions through multi-region "network of networks" models. Curr Opin Neurobiol. 2020;65: 146-151.

35. Glaser JI, Whiteway M, Cunningham JP, Paninski L, Linderman SW. Recurrent Switching Dynamical Systems Models for Multiple Interacting Neural Populations. bioRxiv. 2020. p. 2020.10.21.349282. doi:10.1101/2020.10.21.349282

36. Whittington JCR, Muller TH, Mark S, Chen G, Barry C, Burgess N, et al. The Tolman-Eichenbaum Machine: Unifying Space and Relational Memory through Generalization in the Hippocampal Formation. Cell. 2020;183: 1249-1263.e23.

37. Song HF, Yang GR, Wang X-J. Reward-based training of recurrent neural networks for cognitive and value-based tasks. Elife. 2017;6. doi:10.7554/eLife.21492

38. O'Reilly RC, Frank MJ. Making working memory work: a computational model of learning in the prefrontal cortex and basal ganglia. Neural Comput. 2006;18: 283-328.

39. Logiaco L, Sean Escola G. Thalamocortical motor circuit insights for more robust hierarchical control of complex sequences. arXiv [cs.LG]. 2020. Available: http://arxiv.org/abs/2006.13332

40. Cueva CJ, Wang PY, Chin M, Wei XX. Emergence of functional and structural properties of the head direction system by optimization of recurrent neural networks. arXiv preprint arXiv:191210189. 2019. Available: http://arxiv.org/abs/1912.10189

41. Werbos PJ. Backpropagation through time: what it does and how to do it. Proc IEEE Inst Electr Electron Eng. 1990;78: 1550-1560.

42. Williams RJ, Zipser D. Experimental Analysis of the Real-time Recurrent Learning Algorithm. Conn Sci. 1989;1: 87-111.

43. Murray JM, Escola GS. Learning multiple variable-speed sequences in striatum via cortical tutoring. Elife. 2017;6. doi:10.7554/eLife.26084

44. Marschall O, Cho K, Savin C. A unified framework of online learning algorithms for training recurrent neural networks. J Mach Learn Res. 2020. Available:

https://www.jmlr.org/papers/volume21/19-562/19-562.pdf

45. Pascanu R, Mikolov T, Bengio Y. On the difficulty of training recurrent neural networks. International conference on machine. 2013. Available:

http://www.jmlr.org/proceedings/papers/v28/pascanu13.pdf?source=post_page- 
46. Bengio $Y$, Simard $P$, Frasconi P. Learning long-term dependencies with gradient descent is difficult. IEEE Trans Neural Netw. 1994;5: 157-166.

47. Hochreiter S, Schmidhuber J. Long Short-Term Memory. Neural Comput. 1997;9: 1735-1780.

48. Bai S, Zico Kolter J, Koltun V. An Empirical Evaluation of Generic Convolutional and Recurrent Networks for Sequence Modeling. arXiv [cs.LG]. 2018. Available: http://arxiv.org/abs/1803.01271

49. Pritzel A, Uria B, Srinivasan S, Badia AP, Vinyals O, Hassabis D, et al. Neural Episodic Control. In: Precup D, Teh YW, editors. Proceedings of the 34th International Conference on Machine Learning. PMLR; 2017. pp. 2827-2836.

50. Botvinick M, Ritter S, Wang JX, Kurth-Nelson Z, Blundell C, Hassabis D. Reinforcement Learning, Fast and Slow. Trends Cogn Sci. 2019;23: 408-422.

51. Ke NR, Singh A, Touati A, Goyal A, Bengio Y, Parikh D, et al. Learning Dynamics Model in Reinforcement Learning by Incorporating the Long Term Future. arXiv [stat.ML]. 2019. Available: http://arxiv.org/abs/1903.01599

52. Wang JX. Meta-learning in natural and artificial intelligence. Current Opinion in Behavioral Sciences. 2021;38: 90-95.

53. Hung C-C, Lillicrap T, Abramson J, Wu Y, Mirza M, Carnevale F, et al. Optimizing agent behavior over long time scales by transporting value. Nat Commun. 2019;10: 5223.

54. Kingma DP, Ba J. Adam: A Method for Stochastic Optimization. arXiv [cs.LG]. 2014. Available: http://arxiv.org/abs/1412.6980

55. Hadsell R, Rao D, Rusu AA, Pascanu R. Embracing Change: Continual Learning in Deep Neural Networks. Trends Cogn Sci. 2020;24: 1028-1040.

56. Finn C, Abbeel P, Levine S. Model-Agnostic Meta-Learning for Fast Adaptation of Deep Networks. In: Precup D, Teh YW, editors. Proceedings of the 34th International Conference on Machine Learning. PMLR; 2017. pp. 1126-1135.

57. Masse NY, Yang GR, Song HF, Wang X-J, Freedman DJ. Circuit mechanisms for the maintenance and manipulation of information in working memory. Nat Neurosci. 2019;22: 1159-1167.

58. Ba J, Hinton G, Mnih V, Leibo JZ, lonescu C. Using Fast Weights to Attend to the Recent Past. arXiv [stat.ML]. 2016. Available: http://arxiv.org/abs/1610.06258

59. Tyulmankov D, Yang GR, Abbott LF. Meta-learning local synaptic plasticity for continual familiarity detection. bioRxiv. 2021. Available: https://www.biorxiv.org/content/10.1101/2021.03.21.436287v1.abstract

60. Wang JX, Kurth-Nelson Z, Kumaran D, Tirumala D, Soyer H, Leibo JZ, et al. Prefrontal cortex as a meta-reinforcement learning system. Nat Neurosci. 2018;21: 860-868.

61. Miconi T, Stanley K, Clune J. Differentiable plasticity: training plastic neural networks with backpropagation. In: Dy J, Krause A, editors. Proceedings of the 35th International 
Conference on Machine Learning. PMLR; 2018. pp. 3559-3568.

62. Confavreux B, Agnes EJ, Zenke F, Lillicrap T, Vogels TP. A meta-learning approach to (re)discover plasticity rules that carve a desired function into a neural network. doi:10.1101/2020.10.24.353409

63. Hansen N. The CMA Evolution Strategy: A Comparing Review. In: Lozano JA, Larrañaga P, Inza I, Bengoetxea E, editors. Towards a New Evolutionary Computation: Advances in the Estimation of Distribution Algorithms. Berlin, Heidelberg: Springer Berlin Heidelberg; 2006. pp. 75-102.

64. Musslick S, Cohen JD. Rationalizing Constraints on the Capacity for Cognitive Control. 2020. doi:10.31234/osf.io/vtknh

65. Yang GR, Cole MW, Rajan K. How to study the neural mechanisms of multiple tasks. Curr Opin Behav Sci. 2019;29: 134-143.

66. Duncker L, Driscoll L, Shenoy KV, Sahani M, Sussillo D. Organizing recurrent network dynamics by task-computation to enable continual learning. Adv Neural Inf Process Syst. 2020;33. Available:

https://proceedings.neurips.cc/paper/2020/file/a576eafbce762079f7d1f77fca1c5cc2-Paper. pdf

67. Bourin M, Hascoët M. The mouse light/dark box test. Eur J Pharmacol. 2003;463: 55-65.

68. Yilmaz M, Meister M. Rapid innate defensive responses of mice to looming visual stimuli. Curr Biol. 2013;23: 2011-2015.

69. Robbins $\mathrm{H}$. Some aspects of the sequential design of experiments. Bull Am Math Soc. 1952. Available:

http://citeseerx.ist.psu.edu/viewdoc/download?doi=10.1.1.335.3232\&rep=rep1\&type=pdf

70. Ma WJ, Peters B. A neural network walks into a lab: towards using deep nets as models for human behavior. arXiv [cs.Al]. 2020. Available: http://arxiv.org/abs/2005.02181

71. Lin WC, Delevich K, Wilbrecht L. A role for adaptive developmental plasticity in learning and decision making. Current Opinion in Behavioral Sciences. 2020. pp. 48-54.

doi:10.1016/j.cobeha.2020.07.010

72. Lake BM, Ullman TD, Tenenbaum JB, Gershman SJ. Building machines that learn and think like people. Behav Brain Sci. 2017;40: e253.

73. Musall S, Kaufman MT, Juavinett AL, Gluf S, Churchland AK. Single-trial neural dynamics are dominated by richly varied movements. Nat Neurosci. 2019;22: 1677-1686.

74. Goyal A, Bengio Y. Inductive Biases for Deep Learning of Higher-Level Cognition. arXiv [cs.LG]. 2020. Available: http://arxiv.org/abs/2011.15091

75. Spelke ES, Kinzler KD. Core knowledge. Dev Sci. 2007;10: 89-96.

76. Deng J, Dong W, Socher R, Li L, Kai Li, Li Fei-Fei. ImageNet: A large-scale hierarchical image database. 2009 IEEE Conference on Computer Vision and Pattern Recognition. 2009. pp. 248-255. 
77. Leibo JZ, de Masson d'Autume C, Zoran D, Amos D, Beattie C, Anderson K, et al. Psychlab: A Psychology Laboratory for Deep Reinforcement Learning Agents. arXiv [cs.Al]. 2018. Available: http://arxiv.org/abs/1801.08116

78. Maheswaranathan N, Williams AH, Golub MD, Ganguli S, Sussillo D. Universality and individuality in neural dynamics across large populations of recurrent networks. Adv Neural Inf Process Syst. 2019;2019: 15629-15641.

79. Schrimpf M, Kubilius J, Lee MJ, Ratan Murty NA, Ajemian R, DiCarlo JJ. Integrative Benchmarking to Advance Neurally Mechanistic Models of Human Intelligence. Neuron. 2020;108: 413-423. 